\title{
LARIISA: soluções digitais inteligentes para apoio à tomada de decisão na gestão da Estratégia de Saúde da Família
}

\author{
Field of practice: LARIISA: smart digital solutions to support \\ decision-making in Family Health Strategy management
}

\author{
Raimundo Valter Costa Filho (https://orcid.org/0000-0002-2456-8819) ${ }^{1}$ \\ José Neuman de Souza (https://orcid.org/0000-0002-2980-8877) ${ }^{2}$ \\ Luiz Odorico Monteiro de Andrade (https://orcid.org/0000-0002-3335-0619) ${ }^{3}$ \\ Antônio Mauro Barbosa de Oliveira (https://orcid.org/0000-0002-7898-9440) ${ }^{1}$ \\ Jean-Louis Denis (https://orcid.org/0000-0003-1295-332X) ${ }^{4}$ \\ Luzia Lucélia Saraiva Ribeiro (https://orcid.org/0000-0003-3277-3780) ${ }^{5}$ \\ Kelen Gomes Ribeiro (https://orcid.org/0000-0003-0870-1971) ${ }^{2}$ \\ Daniel Barreto de Andrade (https://orcid.org/0000-0002-2730-4827) ${ }^{6}$ \\ Silas Santiago Lopes Pereira (https://orcid.org/0000-0003-2535-3024) ${ }^{1}$
}

${ }^{1}$ Instituto Federal de

Educação Ciência e

Tecnologia do Ceará (IFC).

$\mathrm{Km} 132$ s/n, Aeroporto.

62800-000 Aracati CE

Brasil.

valter.costa@ifce.edu.br

${ }^{2}$ Universidade Federal do

Ceará (UFC). Fortaleza CE

Brasil.

${ }^{3}$ Fundação Oswaldo Cruz

Ceará (Fiocruz Ceará).

Eusébio CE Brasil.

${ }^{4}$ Université de Montreal.

Montreal QC Canada.

${ }^{5}$ Instituto Atlântico.

Fortaleza CE Brasil

${ }^{6}$ AVICENA ${ }^{\circledR}$. Fortaleza CE

Brasil.

\begin{abstract}
The LARIISA collaborators group has been conducting research and development of technological solutions to support decision-making in health systems since 2009. GISSA, a cloud system resulting from the scientific and technological evolution of the LARIISA project, is among the solutions produced. This paper aims to describe the developing trend of GISSA (, a technological tool supporting the Family Health Strategy in northeastern Brazil, pointing out challenges, paths, and potentialities. This is a descriptive and exploratory study, based on secondary sources from the IBGE, INMET, SINAN, SIM, and SINASC, with quantitative analysis based on machine-learning techniques applied to create digital health microservices. Operating in the northeast and southeast regions, GISSA $\odot$ provides information that qualifies health managers' decision-making process, improving the municipal health system's management.
\end{abstract}

Key words Digital Health, Health Systems, Family Health Strategy, Information Systems, Mobile Health
Resumo O grupo de colaboradores do LARIISA realiza pesquisa e desenvolvimento de soluções tecnológicas para apoio à tomada de decisão em sistemas de saúde desde 2009. Dentre as soluções produzidas está o GISSA ${ }^{\circledR}$, sistema em nuvem resultado da evolução científica e tecnológica do projeto LARIISA. O objetivo do presente artigo é descrever a trajetória de evolução do GISSA ${ }^{\circledast}$, ferramenta tecnológica que apoia a Estratégia de Saúde da Família no nordeste do Brasil, apontando desafios, caminhos e potencialidades. Trata-se de um estudo descritivo e exploratório, baseado em fontes secundárias do IBGE, INMET, SINAN, SIM e SINASC, com análise quantitativa a partir de modelos de aprendizagem de máquina aplicados na criação de microserviços em saúde digital. Operando nas regiões nordeste e sudeste, o GIS$S A^{\circledR}$ disponibiliza informações que qualificam o processo de tomada de decisão de gestores de saúde $e$, consequentemente, contribui para aperfeiçoar a gestão do sistema de saúde municipal.

Palavras-chave Saúde Digital, Sistemas de Saúde, Estratégia Saúde da Família, Sistemas de Informação, Saúde Móvel 


\section{Introdução}

A gestão em saúde requer a tomada de decisões através da melhor combinação dos recursos disponíveis, aprimorando o funcionamento das organizações, estimulando ações eficientes, eficazes e efetivas. No Brasil, as crises econômicas e sociais somadas às transições demográficas, tecnológica e epidemiológica propiciam o tencionamento para formas mais versáteis de gestão ${ }^{1}$. No Sistema Único de Saúde (SUS) isso se desdobra na necessidade de implantação de políticas de saúde inovadoras.

Neste contexto, insere-se a noção de governança, com arranjos institucionais organizados que envolvem diferentes atores, estratégias e procedimentos para gerir, de forma compartilhada e interfederativa, as relações entre estruturas operacionais, com vistas à obtenção de maior interdependência e melhores resultados sanitários e econômicos ${ }^{2}$. O conceito de governança abrange instituições governamentais, mas implica, também, mecanismos de controle informais de caráter não governamental, os quais fazem com que as pessoas e as organizações dentro de sua área de atuação tenham uma conduta determinada, satisfaçam necessidades e respondam às demandas ${ }^{3}$.

Dentre as respostas às demandas, os Sistemas de Informação em Saúde (SIS) constituem ferramentas importantes para o planejamento e a avaliação das políticas de saúde, assim como dos serviços, redes e sistemas de saúde ${ }^{4}$. Conectados a essas necessidades, em 2009, a equipe de pesquisadores do Laboratório de Redes Inteligentes e Integradas em Saúde (LARIISA) desenvolveu um projeto que endereçava requisitos específicos das cinco áreas clássicas de governança em saúde pública: clínica epidemiológica, técnica administrativa e financeira, normativa, gestão compartilhada e gestão do conhecimento ${ }^{5-7}$. Este sistema seria uma solução digital inteligente e integraria tecnologias como Data Warehouse (DW), ontologias $\left(\right.$ mashups $\left.^{8}\right)$ e Data Mining (DM $)^{9,10}$. Pretendia-se modularizar uma plataforma que servisse de base para a construção de inteligência de governança para apoio à tomada de decisão na gestão de sistemas de saúde.

Os conceitos e experiências adquiridas no projeto, então intitulado LARIISA, serviram como ponto de partida para a proposta do sistema GISSA ${ }^{\circledR 11,12}$ criada para atender à gestão inteligente da Estratégia Saúde da Família (ESF), principal estratégia do Ministério da Saúde (MS) para reorientação dos modelos assistenciais em saúde, visando à reorganização das práticas na atenção primária ${ }^{13}$. Diante dos muitos desafios que permeiam a ESF, apontados na literatura ${ }^{14}$, como a baixa valorização política, econômica e social da estratégia; a baixa densidade tecnológica, a fragilidade dos sistemas de apoio diagnóstico e de informação clínica; e os problemas gerenciais, teve-se a atenção despertada para a necessidade de construção de soluções como o sistema GISSA ${ }^{\circledR}$.

Esta solução foi implantada em 2014 com as funcionalidades: geração de dashboards, indicadores, alertas, relatórios, busca semântica, análise de risco e inferências com base em eventos monitorados pelo Sistema de Informações sobre Nascidos Vivos (SINASC), Sistema de Informação de Agravos de Notificação (SINAN), Sistema de Informação sobre Mortalidade (SIM), Sistema de Atenção Básica do Sistema Único de Saúde (eSUS AB), Sistema de Informações do Programa Nacional de Imunizações (SIPNI) e Cadastro Nacional de Estabelecimentos de Saúde (CNES). Uma prova de conceito foi implantada no município de Tauá, Ceará, Brasil, passando a integrar estratégias de saúde pública no semiárido cearense. A AVICENA ${ }^{\circledR}$ e o Instituto Atlântico ${ }^{\circledR}$ também foram parceiros neste projeto.

Agora evoluindo através de experiências de campo, o grupo LARIISA em parceria com a startup em saúde digital AVICENA ${ }^{\circledR}$ passou a desenvolver o produto GISSA ${ }^{\circledR}$ e expandir a utilização do sistema prospectando mais municípios para implantação, a maioria na região Nordeste. Neste processo, a solução digital evoluiu naturalmente para módulos que se comunicam e oferecem as funções percebidas pelo usuário. Nesta perspectiva integrou-se os componentes para aquisição, manipulação e visualização da informação, sendo ontologia, inteligência artificial e, mais recentemente, chatbot, serviços acoplados à plataforma para governança em saúde.

A partir desses elementos, tem-se como objetivo do presente artigo descrever a trajetória de evolução do GISSA ${ }^{\circledR}$, ferramenta tecnológica que apoia a Estratégia de Saúde da Família no nordeste do Brasil, apontando desafios, caminhos e potencialidades. Devido à natureza específica de governança do sistema de saúde pública brasileiro e o nicho de aplicação da pesquisa descrita neste artigo, não foi encontrada publicação que exemplifique estudo nacional ou internacional de sistema semelhante para fins de comparação. 


\section{Métodos}

Estudo quantitativo, descritivo e exploratório, realizado com fontes secundárias de sistemas governamentais como Instituto Brasileiro de Geografia e Estatística (IBGE); Instituto Nacional de Meteorologia (INMET) e sistemas nacionais de informação em saúde como o SINASC, SIM e SINAN.

A implementação do sistema de software seguiu o SCRUM ${ }^{15}$, metodologia de desenvolvimento ágil e incremental baseado no conceito de sprints, subdivisões do período do projeto em intervalos. Esta técnica prevê três atores no processo de desenvolvimento do sistema. O product owner, responsável por melhorar a proposta de valores a ser entregue pelo produto; o Time de desenvolvimento, composto pelos profissionais que fazem o trabalho para entregar as versões com melhorias incrementais a cada sprint; e scrum master, técnico com experiência em desenvolvimento responsável por coordenar soluções às demandas apresentadas ao Time de desenvolvimento e ao product owner, certificando-se de que a equipe siga as práticas e regras do SCRUM.

Para facilitar a comunicação, o método requer três encontros para discutir aspectos do projeto: a reunião diária (daily), que acontece com o objetivo de certificar o que foi feito no dia anterior, identificar o que impede as partes da equipe avançar e a alocação/ordenação das atividades remanescentes; a reunião de revisão, realizada ao final da sprint para apresentar as funcionalidades implementadas; e a reunião de retrospectiva, para rever os erros e acertos da última sprint com vistas a promover o aprendizado da equipe.

O trabalho contemplou dois municípios localizados no Ceará, Brasil, sendo o primeiro localizado na região do sertão dos Inhamuns, categorizado como o segundo maior município do estado em área territorial: Tauá; e o segundo, a capital cearense, situada na região nordeste do país: Fortaleza. Uma prova de conceito foi implantada no município de Tauá, Ceará, Brasil, passando a integrar estratégias de saúde pública no semiárido cearense. Em Fortaleza, um mock -up (protótipo) do gráfico epidemiológico semanal para a dengue é apresentado demonstrando previsão realizada na $25^{\text {a }}$ semana de 2020 .

O estudo baseia-se na descrição da composição de duas classes de microserviços de inteligência artificial: análise de risco e predição de indicadores, visando conhecer e apresentar seus desafios e potencialidades. A análise de risco é calculada para a saúde materno e neonatal/infan- til. Já a predição de indicadores foca na vigilância epidemiológica e, de forma mais específica, no conhecimento sobre o conjunto de fatores determinantes e condicionantes da saúde relacionados às arboviroses, com a finalidade de recomendar medidas de prevenção e controle da dengue.

Para a análise de risco no tocante à saúde materno e neonatal/infantil, buscaram-se dados do SISNAC e do SIM dos municípios cearenses de Caucaia, Quixeramobim, Quixeré, Arneiroz, Pacatuba, Sobral e Tauá no período de 2007 a 2018, possibilitando a construção de modelos de aprendizagem de máquina que identifiquem graus variados de risco para a saúde desses grupos, qualificando a tomada de decisão dos gestores em saúde. De forma análoga, utilizaram-se dados do IBGE, do INMET e do SINAN disponíveis para os municípios de Fortaleza, Caucaia, Quixeramobim, Pacatuba, Sobral e Tauá, coletados no período de 2007 a 2019, aplicados aos modelos de aprendizagem de máquina desenvolvidos é possível predizer, a partir dos dados dessas três fontes, o número de indivíduos infectados por dengue a ser confirmado pelo SINAN nas próximas semanas em dada localidade.

A análise quantitativa desses dados deu-se por meio da utilização dos modelos de aprendizagem de máquina desenvolvidos pelo LARIISA, apresentados a seguir. O estudo foi submetido, através da Plataforma Brasil, ao Comitê de Ética em Pesquisa da Escola de Saúde Pública do Ceará (ESP/CE), e foi aprovada.

\section{Resultados e Discussões}

Os serviços de inteligência em saúde podem ser desenvolvidos por muitas entidades públicas ou privadas, bem como pesquisadores independentes que tenham acesso aos dados em diferentes domínios de análise. Desde imagens a informações que evidenciem contato entre pessoas (contact tracing), estes microdados são analisados por equipes capazes de criar modelos específicos em resposta às demandas operacionais. Baseado na tecnologia REST, o módulo de inteligência do GISSA $^{\circledR}$ dispõe dos serviços de inferência de risco de óbito materno e infantil, bem como de modelos para vigilância epidemiológica aplicáveis às arboviroses.

\section{Arquitetura para Sistemas de Saúde Digital}

Saúde digital ou as tecnologias digitais utilizadas para a saúde tornaram-se um importante 
campo de prática por empregar formas rotineiras e inovadoras de Tecnologia da Informação e Comunicação (TIC) para atender às necessidades de saúde. O termo saúde digital está enraizado na eSaúde (eHealth), definida como "o uso da tecnologia da informação e comunicação em apoio à saúde e serviços relacionados ao campo da saúde". Saúde Móvel (mHealth) é um subconjunto de eHealth e é definido como o "uso de dispositivos móveis sem fio para a saúde". Dentro desse contexto, o termo saúde digital foi introduzido como "um amplo guarda-chuva que engloba eHealth, bem como áreas emergentes, como o uso das ciências avançadas em "big data", genômica e inteligência artificial ${ }^{16}$.

Neste estudo, aborda-se a composição de dois microserviços de inteligência artificial, os quais fazem parte do sistema GISSA ${ }^{\circledR}$, nascido de uma solução para governança de saúde pública na atenção básica e em processo de evolução na atualidade. Mostra-se como ferramenta de apoio à tomada de decisão baseada em informações extraídas dos sistemas nacionais de informação em saúde SINASC, SIM, SINAN, e-SUS, SIPNI e CNES; incluindo outros sistemas governamentais, como IBGE, INMET e Tribunal de Contas do Estado (TCE). Nos trabalhos aqui descritos, os dados são extraídos de bancos de dados municipais e sistemas web de interesse (Figura 1), sendo agregados e estruturados em informações úteis para a equipe de saúde da família e gestão municipal, apoiando políticas públicas. Os serviços de ontologia, inteligência artificial e, mais recentemente, chatbot compõe três blocos distintos que cooperam com o sistema provendo funções específicas via consulta.

Na base dos sistemas de saúde 4.0 estão a fonte dos dados. Cada sistema que fornece informações sobre o indivíduo (microdado) é considerado uma microfonte. Inúmeros são os exemplos na saúde, desde pulseiras eletrônicas (smartbands) às máquinas de processamento reversetranscriptase polymerase chain reaction (RT-PCR) utilizadas para exames de detecção viral, produzindo enorme quantidade de dados todos os dias. Ainda na geração, estes microdados são qualificados e, com o consentimento do indivíduo, gravados no próximo nível da arquitetura, mantendose sob o controle da instituição responsável, seja autoridades de saúde ou empresas especializadas (hospitais, planos de saúde, startups, etc.). Os algoritmos que manipulam os microdados para traduzi-los em informação útil encontram-se na camada de serviços. A título de exemplo, nesta abordagem, um microserviço agregaria casos de COVID-19 registrados no ano 2020, na cidade de Fortaleza, numa tabela intermediária, e outro utilizaria este resultado para apresentar o gráfico de casos por semana epidemiológica à equipe de gestão de saúde na camada de aplicações.

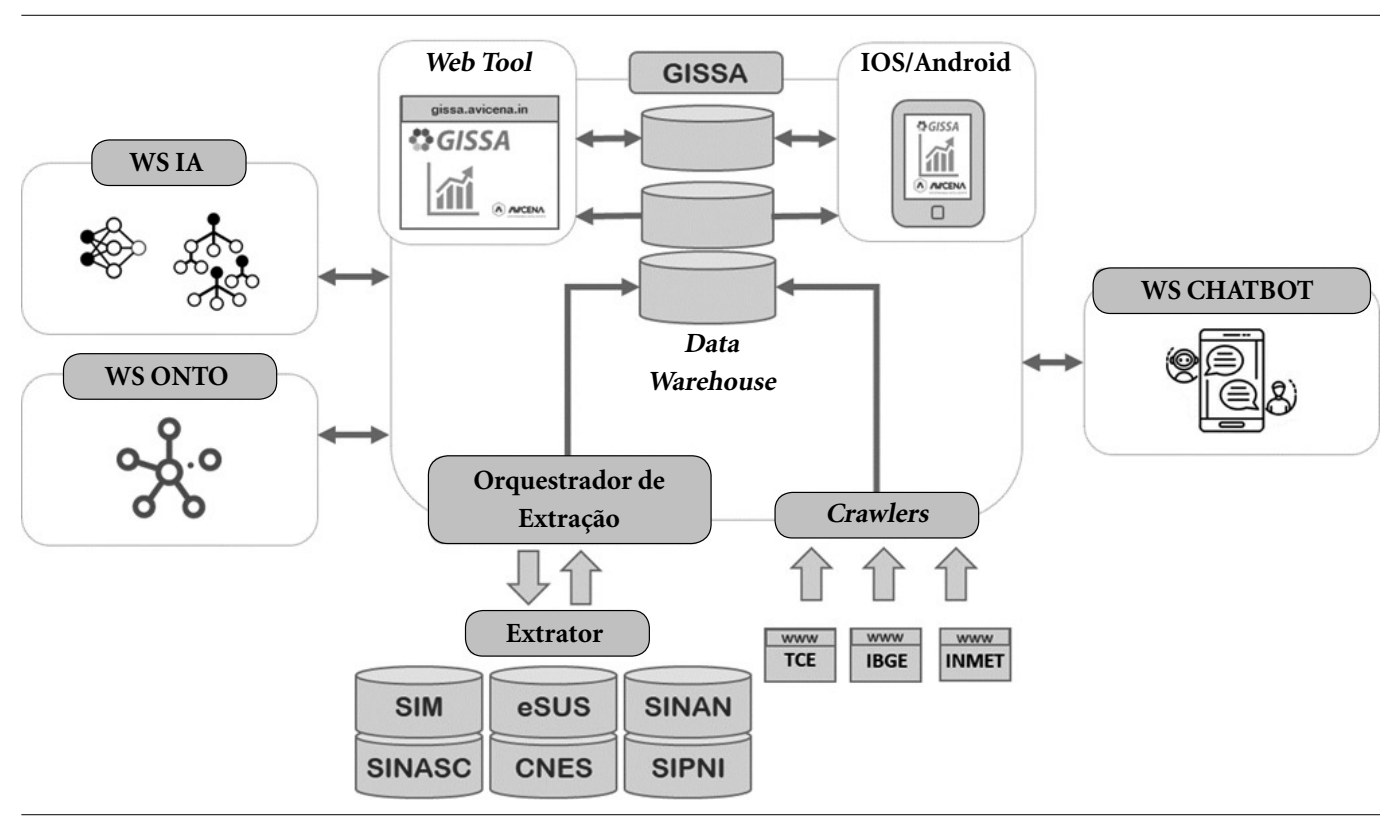

Figura 1. Componentes da arquitetura atual do sistema. 
A Figura 2 apresenta a arquitetura de sistemas de saúde considerando a estrutura de microerviços, identificando os microserviços de inteligência artificial como subconjunto dos serviços implementados nesta camada.

Neste sentido, a Política Nacional de Informação e Informática em Saúde (PNIIS) promovida pelo MS por meio do Departamento de Informática do SUS (DATASUS), propôs, no final de 2019, a Rede Nacional de Dados em Saúde (RNDS) ${ }^{17}$. Esta iniciativa possibilita que diferentes parceiros (públicos/privados) possam cooperar com a RNDS. Isso permitiria que informações relacionadas à saúde do cidadão circulem livremente entre os profissionais envolvidos no cuidado, promovendo eficiência no acompanhamento do paciente. Este passo é essencial no desenvolvimento de sistemas que possam construir a jornada do usuário nas várias linhas de cuidado que acessam as diferentes redes assistenciais do SUS.

Atualmente o sistema GISSA $^{\circledR}$ vive a agregação de novas funções, seguindo uma arquitetura de maior granularidade com a proposta de microserviços em saúde. Neste paradigma de construção de sistemas digitais, um conjunto de serviços atômicos são projetados com a tecnologia Representational State Transfer (REST), promovendo um ambiente de execução estável e o desenvolvimento de aplicações escaláveis. Isto é, atende a um usuário com a mesma qualidade que atende milhões espalhados pelo mundo.

Como um subgrupo destes microserviços estão os modelos de inteligência artificial. Estes algoritmos avaliam os microdados, viabilizando análises novas acerca da saúde de um indivíduo ou população. Os serviços disponíveis atualmente no módulo de inteligência são a análise de risco materno, risco neonatal/infantil e, mais recentemente, a detecção de epidemias produzidas pela arbovirose dengue, projetando o número de casos com semanas de antecedência a serem confirmados pelo SINAN.

\section{Inferência de risco materno e neonatal/infantil}

A coleta de microdados tanto pelo SINASC, ao ocorrer o nascimento de uma criança, quanto pelo SIM, no infortúnio do falecimento, permitem treinar algoritmos para identificar o risco de morte. Mãe (gestante ou puérpera) e filho (neo-

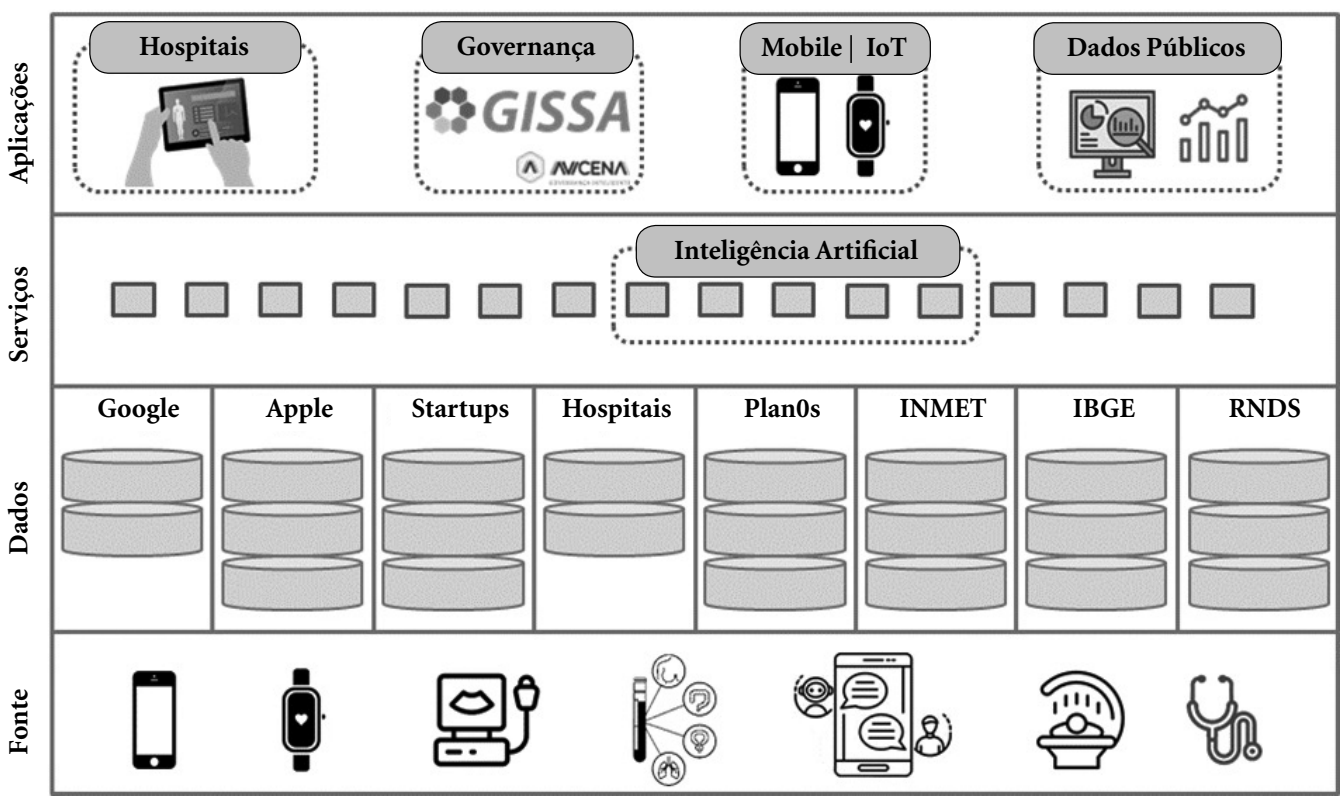

Figura 2. Arquitetura de sistemas de saúde seguindo a utilização de microserviços. 
nato ou infantil) passam a ser assistidos com diferentes modelos de reconhecimento de padrões aplicados em diferentes estágios de gestação e desenvolvimento da criança, oportunizando identificar e prover melhor acompanhamento aqueles casos mais complicados. Além disso, agregar essas informações individualizadas permite que o gestor do sistema de saúde, público ou privado, em diferentes níveis de competência (municipal, estadual ou federal) ou escala, dimensionem a estrutura necessária para atendimento.

Os resultados acadêmicos ${ }^{18-20}$ mostram que é possível identificar o risco de morte materna com parte das informações já coletadas pelo SINASC com mais de $90 \%$ de exatidão a partir de um conjunto de características coletadas pelos formulários de declaração de nascido vivo e óbito. Colaboradores do LARIISA realizaram o cruzamento dos dados coletados nos sistemas de informação de interesse dos municípios da pesquisa entre os meses de janeiro de 2007 a dezembro de 2018, criando dois conjuntos de dados (dataset) distintos, considerando cada contexto de aplicação (materno e neonatal/infantil). Cada amostra corresponde ao conjunto de características da mãe e da criança, que se dividiam em duas classificações: vivos e falecidos (Tabela 1). Os datasets serviram de insumo para o treinamento de modelos de aprendizado de máquina como Gaussian Naive Bayes ${ }^{21}$ (GNB), Random Forest ${ }^{22}$ (RF) e Decision Tree $^{23}$ (DT), que calculam a probabilidade de dado indivíduo pertencer ao grupo com risco de morte. A avaliação de desempenho dos algoritmos de classificação envolveu a aplicação da técnica K-fold Cross Validation $(\mathrm{k}=10)$. A partir da análise dos resultados obtidos, observa-se que os algoritmos chegam a resultados concordantes entre si, tendo o RF obtido maior exatidão dentre os modelos avaliados, provavelmente pela característica deste algoritmo de Ensemble Learning em combinar múltiplas árvores de decisão com o intuito de diminuir a variância e sobreajuste (overfiting), quando o modelo se adapta aos dados usados no treinamento, não classificando corretamente novas amostras.

Tabela 1. Amostras para análise de risco materno e infantil.

\begin{tabular}{lrcc}
\hline \multicolumn{1}{c}{ Dataset } & Vivos & Falecidos & Total \\
\hline Materno & 2.531 & 508 & 3.039 \\
Infantil (0-365 Dias) & 657 & 682 & 1.339 \\
Neonatal (0-28 Dias) & 911 & 952 & 1.863 \\
\hline
\end{tabular}

A Figura 3 demonstra a Receiver Operating Curve (ROC) para o modelo de risco materno que utiliza 18 características - 97,4\% de exatidão - (ex. local do parto, escolaridade da mãe, raça da criança, gênero da criança, etc.) e para o modelo infantil com 27 características - 93,9\% de exatidão - (ex. idade do pai, idade da mãe, escolaridade da mãe, estado civil da mãe, etc. $)^{18}$.

O módulo de inteligência usa classificadores supervisionados de aprendizado de máquina para calcular risco de óbito materno e infantil. Um total de 27 modelos preditivos são gerados para risco de óbito neonatal/infantil e 18 modelos para risco de óbito materno considerando cenários onde subconjuntos de características estão disponíveis para avaliação do risco. A Figura 3 mostra o risco estimado para população atendida pelo sistema de saúde pública na cidade de Tauá, calculado com base nos modelos de análise de risco do sistema GISSA ${ }^{\circledR}$. Os modelos preditivos selecionados para cada cenário de classificação de risco (materno e neonatal/infantil) são serializados e permanecem disponíveis em uma Application Programming Interface (API) REST em nuvem.

De acordo com Canuto $^{24}$, o sistema GISSA ${ }^{\circledR}$ utilizado pelo município de Tauá trouxe visível impacto na dinâmica de trabalho, destacando-se: pactuação de indicadores com dados qualificados; acesso compartilhado, facilitando comunicação; tomada de decisão e corresponsabilidade na produção da saúde com funcionalidade da solução, especialmente alertas e relatórios, colaborando para a construção de uma cultura de planejamento, monitoramento e avaliação em saúde, o que corrobora com as potencialidades do sistema em nuvem estudado.

Das potencialidades, destacam-se também a vontade política e compreensão técnica do gestor e da equipe que recepcionou o sistema; profissionais concursados que participaram do processo de desenvolvimento e implantação e que seguem na gestão municipal; a construção do projeto a partir das necessidades do município e com a participação dos técnicos locais; entrega dos smarthphones para profissionais de saúde e pessoas da comunidade, instrumentalizando e estimulando o uso da solução; apoio, promoção de capacitação e suporte permanente da coordenação local e de consultora do nível central do projeto; articulação do GISSA ${ }^{\circledR}$ com o Projeto Planificação da Atenção Primária à Saúde ${ }^{25}$, que lhe antecedeu em Tauá, com qualificação profissional de toda a força de trabalho em saúde no nível da atenção primária à saúde, qualificando ao mesmo tempo o Sistema de Saúde e o Siste-

Fonte: SINASC e SIM, elaboração dos autores. 

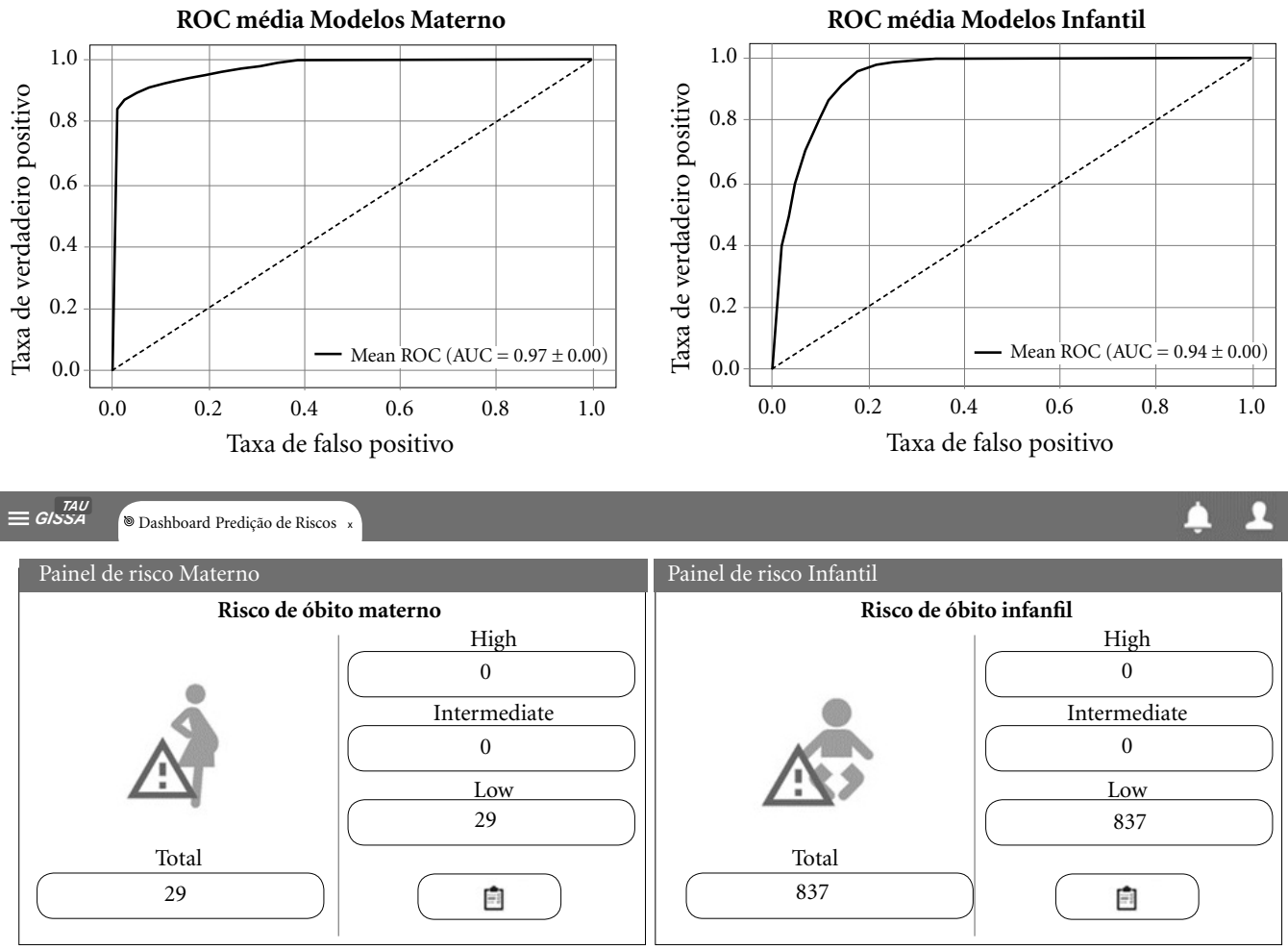

Figura 3. Receiver Operating Curve (ROC) média para os modelos de predição e Tela do GISSA ${ }^{\circledR}$ para acompanhamento de risco de mães e filhos acessado em agosto de 2020.

Fonte: Portal GISSA ${ }^{\circledR}$ (https://gissa.avicena.in/)

ma de Informação; unidades básicas de saúde escolhidas para prova de conceito com acúmulo de experiências exitosas, consideradas "unidades laboratório" no Projeto Planificação e em outras iniciativas; envolvimento da comunidade na prevenção e promoção da saúde, com seleção de gestantes e mães de crianças menores de 2 anos para participar do projeto.

Os desafios também foram evidenciados, como a alternância de poder político, com demonstração de um comprometimento parcial na continuidade do desenvolvimento do projeto GISSA ${ }^{\circledR}$; a substituição de profissionais de enfermagem (coordenadores) nas unidades básicas de saúde, inclusive das unidades da prova de conceito, permanecendo os novos admitidos, com vínculos contratuais também precários; rotatividade de técnicos; falhas técnicas quanto à ausência de sinal de internet e de funcionamento dos robôs do GISSA ${ }^{\circledR}$ para captação de informações dos sistemas de saúde, comprometendo seu uso e desmotivando as equipes; existência de um número significativo de profissionais com limitação quanto ao uso da tecnologia digital, o que nos leva a reflexão sobre a importância do letramento digital ${ }^{26}$, também, dos profissionais da saúde.

\section{Vigilância epidemiológica}

A vigilância Epidemiológica é definida pela legislação ${ }^{27}$ como um conjunto de ações que proporciona o conhecimento, a detecção ou prevenção de qualquer mudança nos fatores determinantes e condicionantes de saúde individual ou coletiva, com a finalidade de recomendar e adotar medidas de prevenção e controle das doenças ou agravos.

No contexto deste estudo, atua-se com uma contribuição num conjunto de ações que proporcionem o conhecimento sobre fatores determinantes e condicionantes de saúde no tocante ao tema das arboviroses. Num país de dimensões 
continentais como o Brasil, onde encontram-se climas bem distintos como o semiárido e o tropical, as arboviroses (dengue, zica, febres chikungunya e amarela) são doenças endêmicas em algumas localidades, mantendo um platô mínimo de casos por ano, podendo, dependendo das condições, evoluir para epidemia ${ }^{28}$.

O nordeste brasileiro tem sofrido bastante principalmente com casos de dengue, forma mais popular de arbovirose circulante nesta região. Por serem doenças correlacionadas e dependentes do mosquito transmissor com potencial de grandes epidemias, o MS acompanha a proliferação do mosquito em áreas urbanas através do Levantamento Rápido de Índices para Aedes Aegypti (LIRAa $)^{29}$. Outra ferramenta fundamental no combate às arboviroses é o SINAN, que disponibiliza microdados do acompanhamento de infectados.

É bastante experimentada a ideia de que a curva de casos de infecção acumulados segue a função matemática logística ou sigmoide, sendo a quantidade de casos por semana obtida pela derivada ${ }^{30}$. Em um evento de potencial epidêmico observa-se o espalhamento viral em quatro fases bem definidas: (I) crescimento exponencial do número de infectados, (II) redução rápida da taxa de novos casos, (III) inversão na taxa de novos casos e (IV) redução exponencial do número de novos casos. Por se tratar de processo estocástico, naturalmente relacionamos diversos fatores correlacionados às epidemias de arboviroses, em especial medidas meteorológicas que influenciam diretamente à proliferação do vetor da doença: o mosquito ${ }^{31,32}$. Medidas populacionais, meteorológicas e de infecção agregadas são subsídios para que um conjunto de Redes Neurais Artificiais (RNA) predigam a quantidade de infectados que serão observados nas semanas subsequentes, o que possibilita recomendar e adotar medidas de prevenção e controle das doenças ou agravos relativos às arboviroses.

As informações relacionadas às arboviroses são mantidas e disponibilizadas pelo INMET $^{33}$, IBGE $^{34}$ e SINAN ${ }^{35}$, todos fazem parte da estrutura de informação do governo federal brasileiro. $\mathrm{O}$ INMET é responsável, dentre outras atribuições, manter e coletar informações meteorológicas de bases espalhadas pelo território nacional. $\mathrm{O}$ IBGE mantém e atualiza a caracterização da população em cada região e o SINAN coleta e disponibiliza o quantitativo infectados por região através do acompanhamento das notificações compulsórias.

Para a criação do microsserviço de predição do número de casos de dengue a serem confirma- dos nas próximas semanas, coletou-se dados das cidades cearenses de Fortaleza, Caucaia, Quixeramobim, Pacatuba, Sobral e Tauá. Ao todo foram observados 65 eventos entre surtos e epidemias distribuídos em 27.895 amostras divididas em dois grupos somando: 9.445 dias atípicos (com 5 ou mais registros confirmados de infecção) e 18.450 dias típicos (menos que 5 casos). As medições foram realizadas diariamente entre janeiro de 2007 a dezembro de 2019. Para cada amostra diária agregaram-se os dados: média móvel do número de infectados por dengue; precipitação chuvosa acumulada (mm); evaporação acumulada $(\mathrm{mm})$; média móvel para temperaturas máxima, média e mínima diárias; média móvel para insolação diária (horas); média móvel para velocidade do vento $(\mathrm{m} / \mathrm{s})$; população e densidade demográfica. Todas as médias móveis e acumulações consideram as medições coletadas nos últimos 7 dias a partir da data da amostra.

Considerando-se surtos ou epidemias com duração mínima de 7 e máxima de 60 semanas e dado a média de duração dos eventos sendo 30 semanas, optou-se por gerar 10 modelos para predição da quantidade de infectados nas semanas futuras $1,2,3,4,5,6,7,8,9$ e 10 . Durante o processo de mineração de dados fica claro o trade off entre exatidão e a capacidade de previsão em semanas futuras. Percebe-se que os modelos de previsão perdem exatidão na medida em que se prevê o comportamento do número de infectados mais semanas à frente. Prevendo a incidência da semana seguinte, observa-se Mean Absolute Error (MAE) de 3,18 infectados por previsão realizada, 4,07 ( $5^{\text {a }}$ semana) e 5,62 (10 a semana).

Outro aspecto observado é que a predição reduz sensibilidade, isto é, perde a capacidade de prever pequenos eventos (abaixo de 60 casos semanais) na medida em que se utiliza dados de cidades com quantidade populacional discrepantes. Amostras de eventos de infecção em Fortaleza (2.7 milhões de habitantes) são facilmente bem maiores que os ocorridos em outras cidades como Caucaia (361 mil), Sobral (210 mil), Pacatuba (83 mil) ou Tauá $(59 \text { mil })^{17}$. Os resultados para a semana 1 (subsequente ao dia de medição) demonstram MAE de 20,35 infectados por previsão realizada, MAE de 31,02 (5a semana) e MAE de 45,39 (10 a semana). Devido a isso, propõe-se dois modelos: cidades pequenas, menores que $150 \mathrm{mil}$ habitantes, e cidades grandes, acima desta marca.

Tomando uma epidemia ocorrida em 2008 na cidade de Fortaleza, a Figura 4 mostra a evolução da predição a partir da $4^{\text {a }}$ semana. Adicionalmente, apresenta-se a tela conceitual do serviço de vi- 

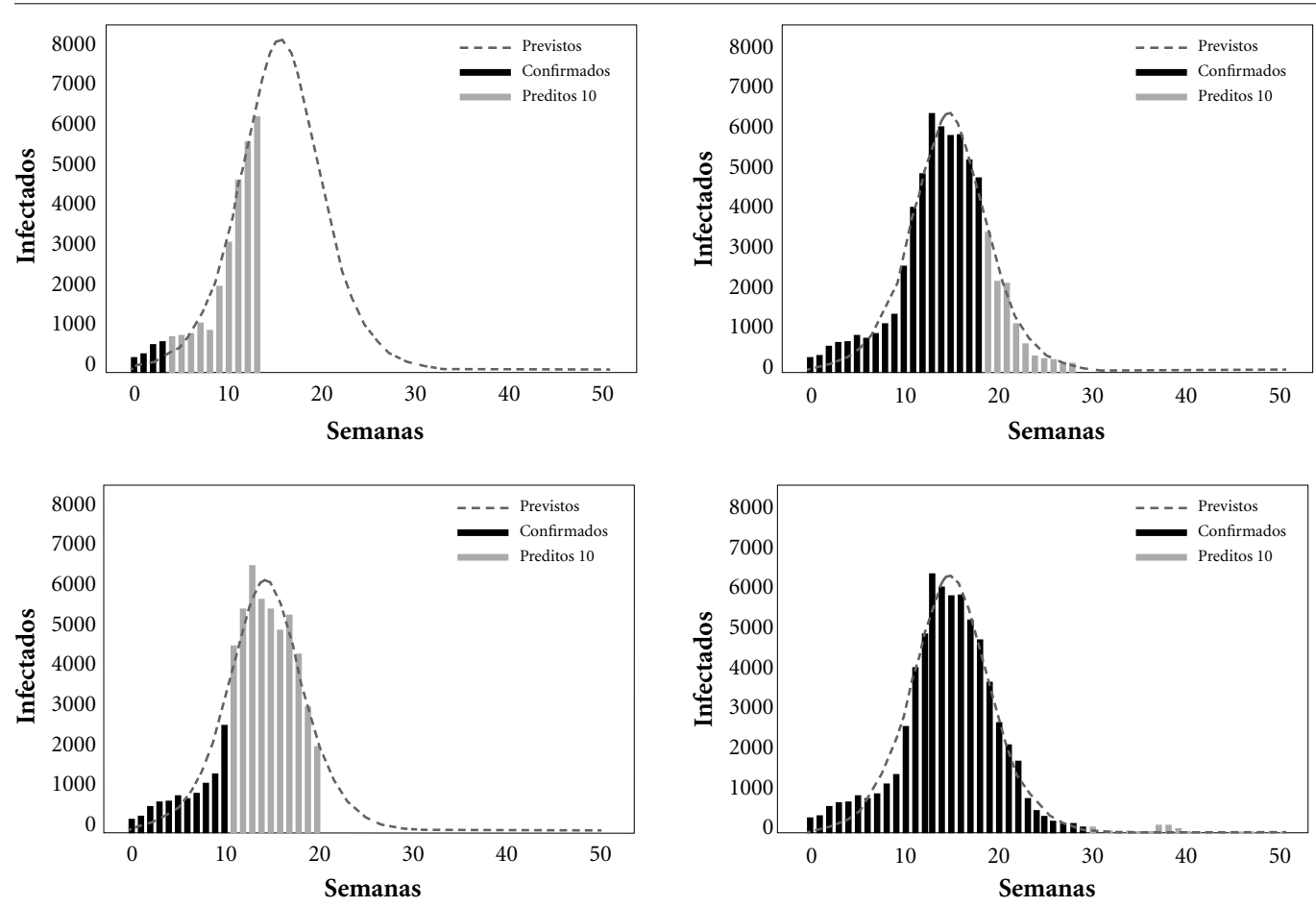

\section{$\equiv G / \triangle S A$} in. Indicadores $x$

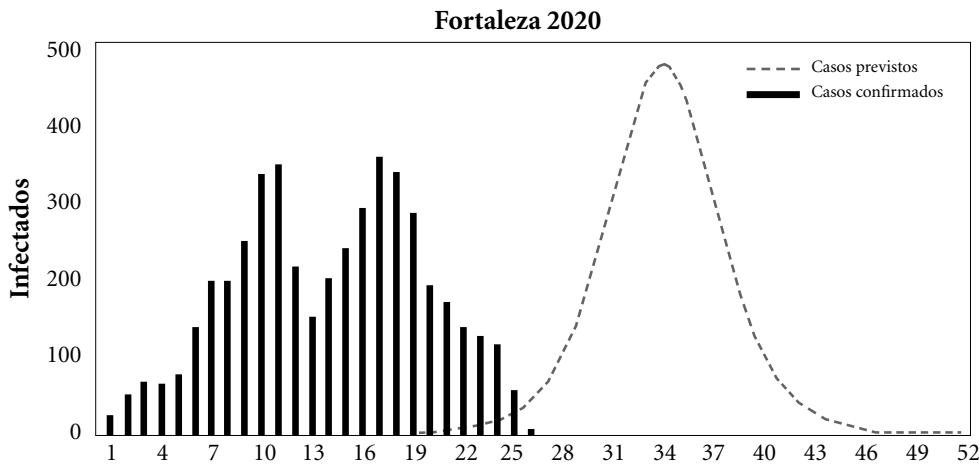

Semana epidemiológica

Figura 4. Evolução da previsão de epidemia ocorrida na cidade de Fortaleza em 2008 e prova de conceito aplicada ao mesmo município na 25a semana de 2020.

Fonte: próprio autor.

gilância epidemiológica para Dengue aplicado ao município de Fortaleza dado medições do ano de 2020. A previsão na $25^{\mathrm{a}}$ semana aponta para um aumento do número de casos nas semanas subsequentes, evidenciando a tendência de surtos locais, ou mesmo epidemia com pico na $34^{\mathrm{a}}$ semana epidemiológica.

Os experimentos apontam que ampliar a predição para mais que 10 semanas incorre em instabilidade e, possivelmente, sombreamento de eventos, isto é, quando duas epidemias de tamanhos diferentes ocorrem com picos de infecção próximos. Este comportamento é mitigado reduzindo o número de predições de incidência de infecção de acordo com as fases da epidemia: 10 semanas a frente para fases I, II e IV; 5 semanas para a fase III.

Ressalta-se que mais experimentos são necessários com o propósito de aferir a sensibilidade da predição para regiões distintas daquelas onde 
o modelo foi concebido e que esta técnica pode ser rapidamente adaptada para outros eventos de arboviroses como febre chikungunaya, febre amarela e Zica, pois dependem do mesmo vetor. Adicionalmente, a modelagem matemática pode ser adaptada a qualquer epidemia (tuberculose, cólera, COVID-19, etc.), bastando escolher variáveis que se correlacionem às condições de presença do vírus em circulação e contaminação.

\section{Considerações Finais}

O GISSA ${ }^{\circledR}$ é um produto comercial resultado da evolução científica e tecnológica do projeto LARIISA. Atualmente é operado no Brasil nas regiões Nordeste e Sudeste, como um sistema em nuvem que disponibiliza informações qualificadas na gestão do sistema de saúde municipal. Estas informações contextualizadas qualificam o processo de tomada de decisão de gestores de saúde no nível municipal.

Adicionalmente aos dados com origem nos sistemas de saúde mantidos pelo governo brasileiro, o sistema coleta diferentes fontes de dados para detecção de contexto e aplicação de técnicas de inteligência artificial. O GISSA ${ }^{\circledR}$ possui uma visão ampliada da gestão de saúde pública, levando em consideração desde aspectos epidemiológicos até financeiros e regulatórios.

A modularização do GISSA ${ }^{\circledR}$ permite que microsserviços de inteligência desenvolvidos possam ser utilizados por diferentes parceiros.
Neste cenário, sistemas distintos consultarão o servidor cognitivo do GISSA ${ }^{\circledR}$, que responde instantaneamente sobre análise de risco e predição de indicadores. Entretanto, para outras aplicações que manipulam maior volume de dados e requerem menor tempo de resposta, maior a velocidade de comunicação necessária, horizonte que se abre com as promessas de conexão do 5G. Outro desafio para popularização de técnicas de inteligência artificial aplicadas em saúde digital é a padronização na representação da informação (imagens, protocolos médicos, designação de doenças, imunização, etc.). Apesar do que já foi realizado, ainda há um caminho longo até garantir interoperabilidade entre os sistemas e cenários futuristas que possam se confirmar para os usuários de sistemas de saúde.

No Brasil, ainda não é popular o uso de sistemas inteligentes relevantes semelhantes ao GIS$\mathrm{SA}^{\circledR}$ para governança de sistema de saúde local ou regional, considerando requisitos específicos de cinco áreas clássicas de governança. Desde a criação do Projeto LARIISA em 2009 até o produto GISSA ${ }^{\circledR}$, já disponível comercialmente e em operação em vários municípios brasileiros, é importante destacar a rica trajetória acadêmica desenvolvida em consonância com o projeto, totalizando duas dúzias de teses e dissertações, mais de quarenta artigos científicos publicados em vários eventos internacionais, muitos protótipos e registros de software, o que motiva a equipe a continuar pesquisando e desenvolvendo contribuições para aperfeiçoar a gestão de sistemas de saúde. 


\section{Colaboradores}

RV Costa Filho, KG Ribeiro, SSL Pereira, DB Andrade e LLS Ribeiro, contribuíram com elaboração do desenho do manuscrito - introdução, arquitetura para sistemas de saúde digital, microsserviços de inteligência artificial, considerações finais. AMB Oliveira e JN Souza contribuíram com a revisão crítica da primeira versão do manuscrito. LOM Andrade e J-L Dennis colaboraram com leitura final e revisão crítica do artigo. Todos os autores aprovaram a versão final e são responsáveis por todos os aspectos deste trabalho, incluindo a garantia de precisão e integridade.

\section{Agradecimentos}

Nossos sinceros agradecimentos à Financiadora de Estudos e Projetos (FINEP), Fundação Cearense de Apoio ao Desenvolvimento Científico e Tecnológico (FUNCAP) por financiarem os projetos de desenvolvimento. Ao apoio da Fundação Oswaldo Cruz (FIOCRUZ) por meio do programas como Inova Fiocruz. Aos pesquisadores do grupo LARIISA, em especial à Dra. Ivana Cristina de Holanda Cunha Barreto, César Olavo de Moura Filho e às instituições parceiras AVICENA ${ }^{\oplus} \mathrm{e}$ Instituto Atlântico ${ }^{\circledast}$.

\section{Referências}

1. Dermindo MP. Gestão eficiente na saúde pública brasileira. JMPHC 2019; 11(Supl. 1):e55s.

2. Mendes EV. As redes de atenção à saúde. Belo Horizonte: Escola de Saúde Pública; 2012.

3. Andrade LOM. Inteligência de Governança para apoio à Tomada de Decisão. Cien Saude Colet 2012; 17(4):829-837.

4. Ferla AA, Cecim RB, Alba RD. Information, education and health care work: Beyond evidence, collective intelligence. RECIIS 2012; 6(2):1-13

5. Oliveira M, Hairon C, Andrade O, Moura R, Sicotte C, Denis J-L, Fernandes S, Gensel J, Bringel J, Martin H. A context-aware framework for health care governance decision-making systems: A model based on the brazilian digital $t v$. In: 2010 IEEE International Symposium on "A World of Wireless, Mobile and Multimedia Networks" (WoWMoM); 2010. p. 1-6.

6. Denis J-L, Champagne F, Pomey M. Towards a framework for analysis of governance in health care organisations and systems. Searching Publications - CCHSA; 2008.

7. Young GJ, Meterko M, Beckman H, Baker E, White B, Saut KM, Greene R, Curtin K, Bokhour B, Berlowitz D, Burgess J. Effects of paying physicians based on their relative performance for quality. J Gen Intern Med 2007; 22(6):872-876.

8. Silva BM, Rodrigues JJ, Díez IT, Coronado ML, Saleem K. Mobile-health: A review of current state in 2015. J Biomed Inform 2015; 56:265-272.

9. Dey AK, Abowd GD, Salber D. A conceptual framework and a toolkit for supporting the rapid prototyping of context-aware applications. Hum Comput Interact 2001; 16(2-4):97-166.

10. Moudani W, Hussein M, Razzak MA, Camino FM. Heart disease diagnosis using fuzzy supervised learning based on dynamic reduced features. Int J E Health Med Commun 2014; 5(3):78-101.

11. Oliveira M, Andrade LOM, Ramos R. Projeto GISSA: Meta física 3 - Atividade 3.1: definir modelo de inteligência de gestão na saúde. Instituto Atlântico; 2016.

12. Gardini LM, Braga R, Bringel J, Oliveira C, Andrade R, Martin H, Andrade LOM, Oliveira M. Clariisa, a context-aware framework based on geolocation for a health care governance system. In: 2013 IEEE 15th International Conference on e-Health Networking, Applications and Services (Healthcom 2013); 2013. p. 334-339.

13. Andrade LOM., Barreto ICHC. Ribeiro KG, Uchoa AAC. A Estratégia Saúde da Família e o SUS. In: Rouquayrol MZ. Epidemiologia \& Saúde. 8ª ed. Rio de Janeiro: MedBook; 2018.

14. Mendes EV. O cuidado das condições crônicas na atenção primária à saúde: o imperativo de consolidação da estratégia da saúde da família. Brasília: OPAS; 2012.

15. Sutherland J, Schwaber K. The Scrum Papers: Nut, Bolts, and Origins of an Agile Framework. Scrum Inc.; 2011.

16. World Health Organization (WHO). WHO guideline: recommendations on digital interventions for health system strengthening. Geneva: WHO; 2019.

17. Brasil. Ministério da Saúde (MS). Conecte SUS avança em todo país com a implantação da rede nacional de dados em saúde [Internet]. 2020 [acessado 2020 jan 23]. Disponível em: https://www.saude.gov.br/noticias/agencia-saude. 
18. Valter R, Santiago S, Ramos R, Oliveira M, Andrade LOM, Barreto ICHC. Data mining and risk analysis supporting decision in brazilian public health systems. In: IEEE International Conference on E-health $\mathrm{Ne}$ tworking, Application Services (HealthCom), Bogotá, Colombia; 2019. p. 1-6.

19. Ramos RF, Mattos CLC, Souza Junior AH, Rocha Neto AR, Barreto GA, Mazzal HA, Mota MO. Heart diseases prediction using data from health assurance systems in models and methods for supporting decision-making in human health and environment protection. In: Computer Systems and Applications (AICCSA), Nova Publishers; 2016. p. 1-5.

20. Nascimento LFC, Rizol PMSR, Abiuzi LB. Establishing the risk of neonatal mortality using a fuzzy predictive model. Cad Saude Publica 2009; 25(9):2043-2052.

21. Witten IH, Eibe F, Hall MA. Data Mining: Practical machine learning tools and techniques. São Francisco: Morgan Kaufmann Pub; 2005.

22. Hastie T, Tibshirani R, Friedman J. The Elements of Statistical Learning. New York: Springer New York Inc; 2001.

23. Liu Y, Wu H. Water bloom warning model based on random forest. In: International Conference on Intelligent Informatics and Biomedical Sciences (ICIIBMS) IEEE; 2017. p. 45-48.

24. Canuto OMC. Relatório de Impacto Socioeconômico do Projeto Gissa, em Prova de Conceito, no Município de Tauá, Ceará, Período 2016 a 2018. Fortaleza: Planejasus; 2018.

25. Brasil. Conselho Nacional de Secretários de Saúde (CONASS). Planificação da Atenção Primária à Saúde nos Estados. Brasília: CONASS; 2011.

26. Cardoso JB. Letramento digital, tecnologias digitais da informação e comunicação e as perspectivas de desenvolvimento social [dissertação]. Itajubá: Universidade Federal de Itajubá; 2020.

27. Brasil. Lei no 8.080, de 19 de setembro de 1990. Dispõe sobre as condições para a promoção, proteção e recuperação da saúde, a organização e o funcionamento dos serviços correspondentes e dá outras providências. Diário Oficial da União 1990; 20 set.

28. Lopes N, Nozawa C, Linhares CRE. Características gerais e epidemiologia dos arbovírus emergentes no Brasil. Rev Pan-Amaz Saude Ananindeua 2014; 5(3):55-64.
29. Brasil. Ministério da Saúde (MS). Secretaria de Vigilância em Saúde. Diretoria Técnica de Gestão. Diagnóstico Rápido nos Municípios para Vigilância Entomológica do Aedes aegypti no Brasil - LIRAa: metodologia para avaliação dos índices de Breteau e Predial. Brasília: MS, 2005.

30. Batista M. Estimation of the final size of the covid-19 epidemic. medRxiv 2020. [preprint] doi:10.1101/2020 .02 .16 .20023606

31. Correia WLFA. Influence of meteorological variables on dengue incidence in the municipality of Arapiraca, Alagoas, Brazil. Rev Soc Bras Med Tropical 2017; 50(3):309-314.

32. Zhao N, Charland K, Carabali M, Nsoesie E, MaherGiroux M, Rees E, Yuan M, Balaguera CG, Ramirez GJ, Zinszer K. Machine learning and dengue forecasting: Comparing random forests and artificial neural networks for predicting dengue burdens at the national sub-national scale in Colombia. PLOS Neglected Tropical Diseases 2020; 14(9):e0008056.

33. Instituto Nacional de Meteorologia (INMET) [Internet]. 2020 [acessado 2020 mar 24]. Disponível em: https://www.inmet.gov.br.

34. Instituto Brasileiro de Geografía e Estatística (IBGE) [Internet]. 2020 [acessado 2020 mar 24]. Disponível em: https://www.ibge.gov.br.

35. Sistema de Informação de Agravos de Notificação (SINAN) [Internet]. 2020 [acessado 2020 mar 24]. Disponível em: https://sinan.saude.gov.br.

Artigo apresentado em 07/08/2020

Aprovado em 18/02/2021

Versão final apresentada em 20/02/2021

Editores-chefes: Romeu Gomes, Antônio Augusto Moura da Silva 